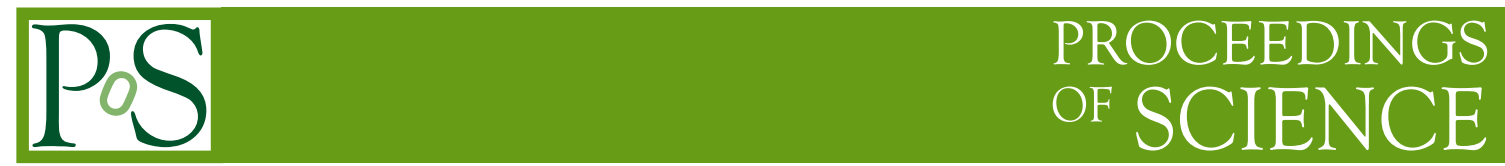

\title{
A brief walk through: Current status of experimental jet physics
}

\section{Eliane Epple}

Los Alamos National Lab,

Physics Division, Los Alamos NM, USA

E-mail: eliane.epple@cern.ch

This is a short summary of the experimental jet results presented during the Hard Probes 2020 conference. I want to highlight that jets are a versatile and powerful probe of the hot and dense QCD medium created in heavy-ion collisions with many approaches that are being explored to learn more about the medium and QCD in general.

HardProbes 2020

1-6 June 2020

Austin, Texas 


\section{Why jets are useful probes}

After decades of studying the hot and dense phase of nuclear matter, which is produced in ultrarelativistic heavy-ion collisions, there still remain many open questions. It remains unclear what type of particles or quasi-particles form the degrees of freedoms throughout the collision phase. It is unclear under which circumstances a hot and dense phase is formed, e.g. in pp collisions. To reveal properties of the medium that is created in these collisions we often study hard probes that traverse the medium. We have seen ample evidence that the properties of those probes are modified by the medium, but we still struggle to understand what the exact mechanisms are that cause these modifications. How does a probe loose energy when it traverses the medium? What properties (color charge, mass etc.) influence the strength of coupling to the medium and thus increase or decrease the energy loss the probe experiences. If the probes are extended, like jets, we can ask what the resolution scale of the medium is. Another question that is of general interest in QCD physics is how the transition from partonic to hadronic degrees of freedom, for example in a jet formation, develops. These and many more open questions are studied in contemporary heavy-ion physics programs.

To answer these open questions various probes are used, among them jets. Jets are particularly useful as probes of the medium since they have many valuable properties other probes lack. Jets have a substructure, thus, they can be used to probe for example effects of color coherence. They cover large kinematic ranges from low to high $p_{\mathrm{T}}$. With tagging methods one can separate quark from gluon jets and light from heavy-quark jets. This unique range and flexibility of jets as probes opens many avenues to study the hot and dense phase of nuclear matter and answer the above discussed, open questions.

\section{What we have learned about jet production so far}

In the following, I want to only briefly summarize some of the current findings of jet studies. For detailed reviews see e.g. Refs. [1-7]. Nowadays, jets are used in multiple approaches as probes of QCD in vacuum and the medium. Based on these studies, we have seen the following picture emerge: if jets are formed in the medium, their average properties change as compared to their vacuum counter parts. We have observed in multiple measurements that their $p_{\mathrm{T}}$ spectrum is shifted causing a visible jet $R_{A A}$ suppression in heavy-ion collisions [8-11]. Multiple experiments have shown that the jets measured in the medium are softer [39], that the hard core of jets gets narrower [38] and that the soft part of the jet structure diffuses to larger angles [40].

\section{Ongoing research}

The effect of a suppression of the nuclear modification factor $R_{A A}$ of jets is well established. One question that is currently under scrutiny is to establish to which extent the jet $R_{A A}$ depends on the selected jet radius, R. Several previous experiments could not find any strong correlation of the jet $R_{A A}$ and jet $\mathrm{R}[8,10]$. A new measurement of CMS that reports the largest set of $R_{A A}$ vs. $\mathrm{R}$ data to date shows some vague hints that the supression gets less with increasing $\mathrm{R}$ [12]. More importantly, this work showcased the importance of such a measurement in general due to 


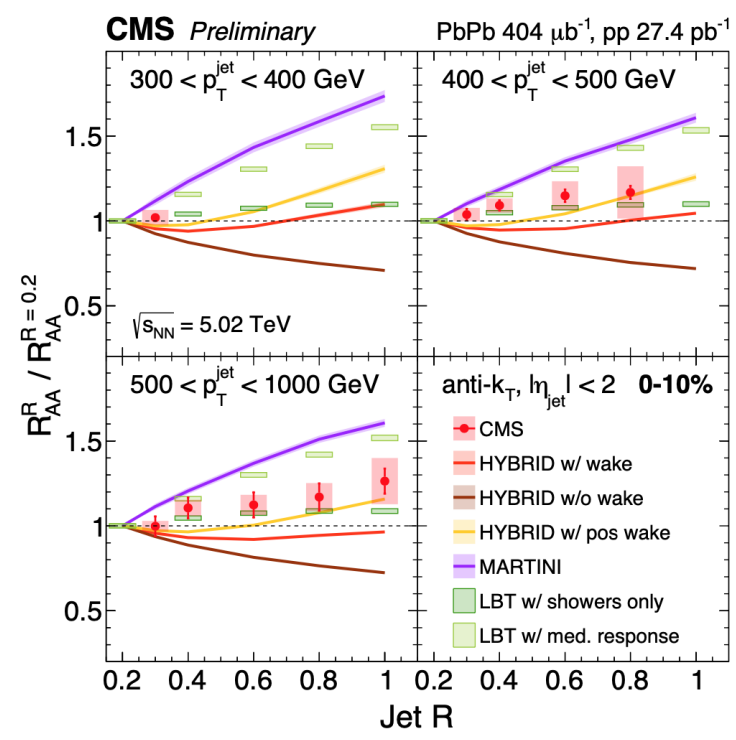

Figure 1: Figure from Ref. [12]. Shown here is the ratio of two $\mathrm{R}_{A A}$ values vs. $\mathrm{R}$ for a given $p_{\mathrm{T}}^{\mathrm{jet}}$. An example selection of theory predictions shows a huge spread of values. For more examples, see the original publication.

its great ability to distinguish the validity of theory predictions, see one example in Fig. 1. The obvious challenge of such measurements is the extension of the jet radius to large values. Two novel approaches are trying to address this. One reclusters smaller radius jets into a larger jet with radius $\mathrm{R}=1$ [13], the other uses machine learning methods to try to subtract the large contibutions of the underlying event in large- $R$ jets [30].

Another open question that is under investigation is the observation of some form of jet suppression in $\mathrm{p}+\mathrm{p}$ and $\mathrm{p}+\mathrm{A}$ collisions. So far, no signs thereof have been reported. During this conference two studies were shown that search for such a signal. One uses trigger-recoil jets in high multiplicity $\mathrm{p}+\mathrm{p}$ collisions [32], the other uses cluster-recoil jets in $\mathrm{p}+\mathrm{A}$ collisions of different event activities [31]. Yet, no signatures deviating from PYTHIA simulations have been found.

A broad class of jet observables focuses on jet substructures and their modification in the medium. For such studies the jets are iteratively declustered and often groomed, see Refs. [33, 34] for details on such techniques. In the vacuum such obtained splittings can be used to compare the splitting phase space in a very meticulous manner to theoretical models [44]. In the medium this splitting phase space can be explored to find regions of modification for the splittings. New in this branch of jet physics are fully unfolded distributons of $\mathrm{z}_{g}$ and $\theta_{g}$ in $\mathrm{A}+\mathrm{A}$ collisions $[14,15]$. No significant modification of the $\mathrm{z}_{g}$ distribution was observed while the opening angle between the subjets shows an enhancement at small values and suppression at large values, see Fig. 2. Another analysis that studies jet splitting is the comparison of $\mathrm{D}^{0}$ tagged to inclusive jets. This shows a suppression of small angle and low number $\left(n_{S D}\right)$ splittings in heavy-flavor jets as compared to inclusive jets $[16,17]$. This is called the "dead cone" effect and is with these measurements now also documented in hadron-hadron collisions.

A third class of jet observables explores the hadronic structure of jet. This is the last stage of jet 

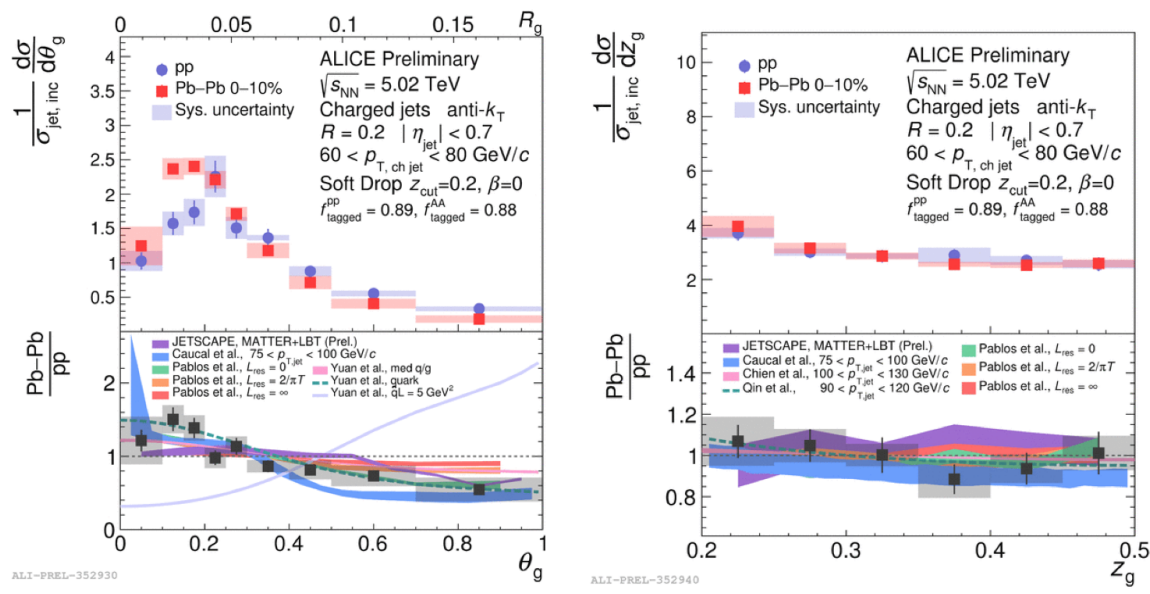

Figure 2: $\theta_{g}$ and $\mathrm{z}_{g}$ distribution and ratios of unfolded jet spectra. Shown is the ratio of $\mathrm{Pb}+\mathrm{Pb}$ to $\mathrm{pp}$ which reveals a supression of large angle splittings and an enhancement of small angle splittings in $\mathrm{Pb}+\mathrm{Pb}$ collisions as compared to pp collisions. The comparision of the $\mathrm{z}_{g}$ distribution shows no evidence for a modification in the momentum balance of the splitting $[14,15]$.

formation after fragmentation and hadronisation. Observables in this class include measurements of momentum fractions in the jet, the radial distribution of jet fragments, and the momentum density of hadrons in radial distance. A recent measurement compared the hadronic structure of b-tagged jets to inclusive jets [18]. The data show that b-jets contain more charged particle tracks at low $\Delta r$ (radial distance to the jet axis) than inclusive jets but also that their overall momentum density extents wider than in inclusive jets. Another kind of jet tagging is done with electro-weak bosons as jet recoil. There are several measurements of $\gamma$ and Z-recoil jet that were published in the last couple years. This tagging enriches the jet sample with light-quark jets and depletes the contribution of gluon-jets. $\mathrm{Z}$ and $\gamma$-tagged jets are harder and more collimated than inclusive jets $[19,20]$. With preselecting the jet type by this sort of tagging, one can study jet modification in the medium in a more controlled manner. Two new results studied the medium modification of the hadronic structure of jets with $Z^{0}$ and $\gamma$-recoil jets [21,35]. This approach ensures, on the one hand, that the jets are calibrated since the boson properties are not modified by the medium and, on the other hand, guarantees that observed effects are not due to an altered mixture of quark and gluon jets, since it is preselecting the former jet type. Figure 3 shows the modification of these jets in central $\mathrm{Pb}+\mathrm{Pb}$ collisions. The left panel shows that the momentum fractions of the jet constituents is suppressed at high values and enhanced at low values. This effect has been observed for inclusive jets before $[41,42]$ but it could not be excluded that it is caused by a change of quark and gluon jet ratio in the medium rather than an intrinsic modification of the jets [36]. The right panel of Fig. 3 shows the $p_{\mathrm{T}}$ density $\rho$ as a function of the distance to the jet axis. One can see again the pattern that the momentum distribution in jets measured in the medium is shifted farther away from the jet axis, effectively broadening the entire jet.

A last, very interesting topic is the measurement of the fragmentation functions of identified hadrons within jets. This should, in the big picture, reveal more about the mechanisms of hadro-

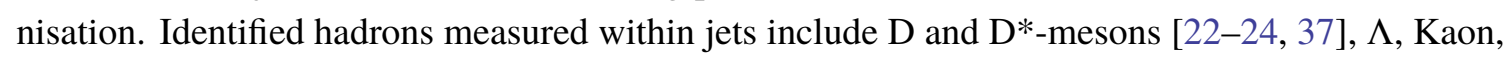





Figure 3: The two panels show the modification of the hadronic structure of jets in $\mathrm{Pb}+\mathrm{Pb}$ collisions as compared to pp collisions. Left, the modification of the framgentation function of Z-boson recoil jets for different centralities [21]. Right, the modification of the momentum density as a function of the distance to the jet axis for $\gamma$ recoil jets [35].

$\Xi$ and $\Lambda_{c}^{+}$[43] and also $J / \psi$-mesons [25-29]. These detailed studies can reveal a lot about our understanding of the formation of these hadrons. Figure 4 illustrates this well. This measurement. among several before [25, 26, 29], shows that the production mechanism of the $J / \psi$-mesons is not modeled correctly in PYTHIA. This accounts specifically for those mesons that do not come from a B-decay. The need to understand this discrepancy becomes more evident if one considers the result of Ref. [28]. In this measurement, $85 \% \pm 3$ (stat) \pm 7 (syst) of the reconstructed $J / \psi$-mesons stemmed from jets. It underlines that an understanding of $J / \psi$-meson production in general is based on an understanding of $J / \psi$-meson production within jets. The analysis of Ref. [29] goes one step further and compares the fragmentation function of $J / \psi$-mesons in jets in pp and $\mathrm{Pb}+\mathrm{Pb}$ collisions. Figure 4, right shows this comparison. The fragmentation function shows a general suppression of high values, $z>0.3$. As this is the limit of the measurement, one can compare these values only to e.g. the first two bins in Fig. 3 left which shows good agreement. Considering that $J / \psi$-mesons are the best studied identified-jet-fragment to date and reveal a lot of surprises, it motivates to search further to what extend we can describe other fragments of the jet.

\section{References}

[1] R. Kogler, B. Nachman, A. Schmidt, L. Asquith, M. Campanelli, C. Delitzsch, P. Harris, A. Hinzmann, D. Kar, C. McLean, J. Pilot, Y. Takahashi, N. Tran, C. Vernieri, M. Vos and E. Winkels, Rev. Mod. Phys. 91 (2019) no.4, 045003 doi:10.1103/RevModPhys.91.045003 [arXiv:1803.06991 [hep-ex]].

[2] J. P. Blaizot and Y. Mehtar-Tani, Int. J. Mod. Phys. E 24 (2015) no.11, 1530012 doi:10.1142/S021830131530012X [arXiv:1503.05958 [hep-ph]].

[3] S. Cao and X. N. Wang, [arXiv:2002.04028 [hep-ph]]. 

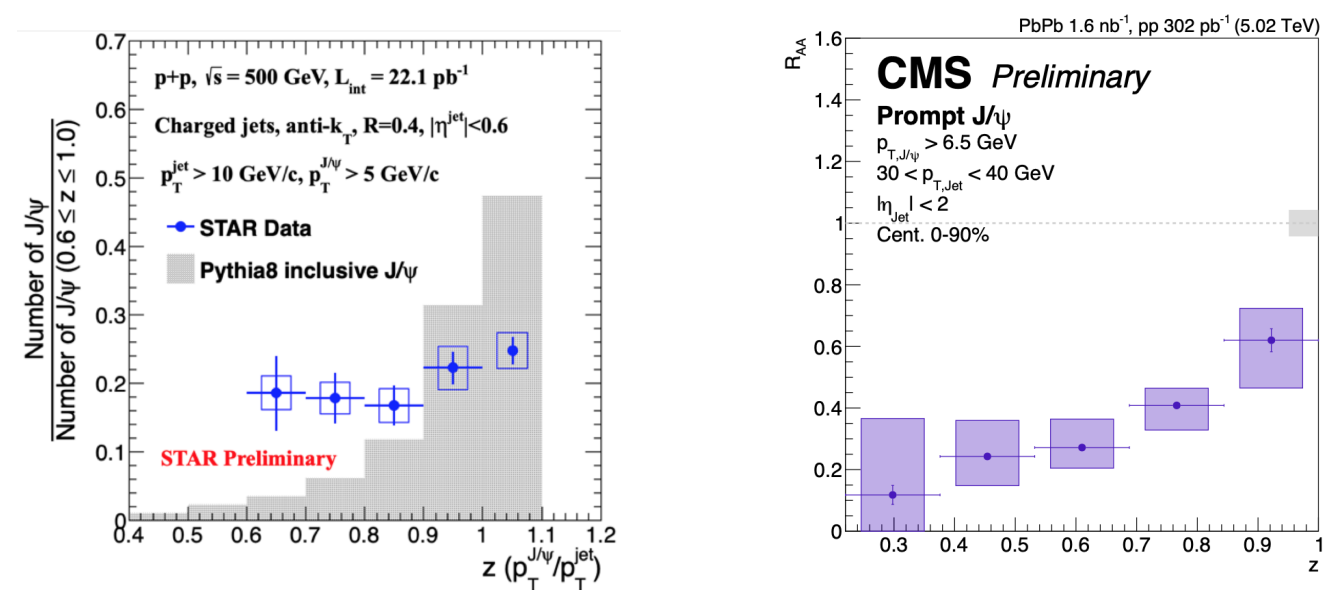

Figure 4: Left, a new measurement of the $J / \psi$-meson fragmentation function in jets. The data are compared to PYTHIA simulations which show a lack of understanding in the hadronisation process, see Ref. [27]. Right, a new meaurment of the nuclear modification of the $J / \psi$-meson fragmentation function in jets. The data show a supression of the fragmentation function in $\mathrm{Pb}+\mathrm{Pb}$ as compared to $\mathrm{pp}$ [29], comparable to the ones in EW-Boson tagged jets shown above.

[4] S. Sapeta, Prog. Part. Nucl. Phys. 89 (2016), 1-55 doi:10.1016/j.ppnp.2016.02.002 [arXiv:1511.09336 [hep-ph]].

[5] M. Connors, C. Nattrass, R. Reed and S. Salur, Rev. Mod. Phys. 90 (2018), 025005 doi:10.1103/RevModPhys.90.025005 [arXiv:1705.01974 [nucl-ex]].

[6] A. J. Larkoski, I. Moult and B. Nachman, Phys. Rept. 841 (2020), 1-63 doi:10.1016/j.physrep.2019.11.001 [arXiv:1709.04464 [hep-ph]].

[7] W. Busza, K. Rajagopal and W. van der Schee, Ann. Rev. Nucl. Part. Sci. 68 (2018), 339-376 doi:10.1146/annurev-nucl-101917-020852 [arXiv:1802.04801 [hep-ph]].

[8] S. Acharya et al. [ALICE], Phys. Rev. C 101 (2020) no.3, 034911 doi:10.1103/PhysRevC.101.034911 [arXiv:1909.09718 [nucl-ex]].

[9] M. Aaboud et al. [ATLAS], Phys. Lett. B $\mathbf{7 9 0}$ (2019), 108-128 doi:10.1016/j.physletb.2018.10.076 [arXiv:1805.05635 [nucl-ex]].

[10] J. Adam et al. [STAR], [arXiv:2006.00582 [nucl-ex]].

[11] V. Khachatryan et al. [CMS], Phys. Rev. C 96 (2017) no.1, 015202 doi:10.1103/PhysRevC.96.015202 [arXiv:1609.05383 [nucl-ex]].

[12] [CMS], CMS-PAS-HIN-18-014.

[13] [ATLAS], ATLAS-CONF-2019-056.

[14] J. Mulligan [ALICE], these proceedings

[15] R. Ehlers [ALICE], these proceedings 
[16] N. Zardoshti [ALICE], Nucl. Phys. [arXiv:2004.05968 [hep-ex]].

[17] V. Kucera [ALICE], these proceedings

[18] A. M. Sirunyan et al. [CMS], [arXiv:2005.14219 [hep-ex]].

[19] R. Aaij et al. [LHCb], Phys. Rev. Lett. 123 (2019) no.23, 232001 doi:10.1103/PhysRevLett.123.232001 [arXiv:1904.08878 [hep-ex]].

[20] M. Aaboud et al. [ATLAS], Phys. Rev. Lett. 123 (2019) no.4, 042001 doi:10.1103/PhysRevLett.123.042001 [arXiv:1902.10007 [nucl-ex]].

[21] [ATLAS], ATLAS-CONF-2019-052.

[22] G. Aad et al. [ATLAS], Phys. Rev. D 85 (2012), 052005 doi:10.1103/PhysRevD.85.052005 [arXiv:1112.4432 [hep-ex]].

[23] S. Acharya et al. [ALICE], JHEP 08 (2019), 133 doi:10.1007/JHEP08(2019)133 [arXiv: 1905.02510 [nucl-ex]].

[24] A. M. Sirunyan et al. [CMS], Phys. Rev. Lett. 125 (2020), 102001 doi:10.1103/PhysRevLett.125.102001 [arXiv:1911.01461 [hep-ex]].

[25] R. Aaij et al. [LHCb], Phys. Rev. Lett. 118 (2017) no.19, 192001 doi:10.1103/PhysRevLett.118.192001 [arXiv:1701.05116 [hep-ex]].

[26] [CMS], CMS-PAS-HIN-18-012.

[27] Q. Yang [STAR], these proceedings

[28] A. M. Sirunyan et al. [CMS], Phys. Lett. B $\mathbf{8 0 4}$ (2020), 135409 doi:10.1016/j.physletb.2020.135409 [arXiv:1910.01686 [hep-ex]].

[29] [CMS], CMS-PAS-HIN-19-007.

[30] H. Bossi [ALICE], [arXiv:2009.02269 [nucl-ex]].

[31] D. Stewart [STAR], these proceedings

[32] F. Krizek [ALICE], these proceedings

[33] F. A. Dreyer, G. P. Salam and G. Soyez, JHEP 12 (2018), 064 doi:10.1007/JHEP12(2018)064 [arXiv:1807.04758 [hep-ph]].

[34] A. J. Larkoski, S. Marzani, G. Soyez and J. Thaler, JHEP 05 (2014), 146 doi:10.1007/JHEP05(2014)146 [arXiv:1402.2657 [hep-ph]].

[35] A. M. Sirunyan et al. [CMS], Phys. Rev. Lett. 122 (2019) no.15, 152001 doi:10.1103/PhysRevLett.122.152001 [arXiv:1809.08602 [hep-ex]]. 
[36] M. Spousta and B. Cole, Eur. Phys. J. C 76 (2016) no.2, 50 doi:10.1140/epjc/s10052-0163896-0 [arXiv:1504.05169 [hep-ph]].

[37] A. I. Sheikh [ALICE], Nucl. Part. Phys. Proc. 309-311 (2020), 174-180 doi:10.1016/j.nuclphysbps.2019.11.030 [arXiv:2009.02751 [nucl-ex]].

[38] S. Acharya et al. [ALICE], JHEP 10 (2018), 139 doi:10.1007/JHEP10(2018)139 [arXiv: 1807.06854 [nucl-ex]].

[39] M. Aaboud et al. [ATLAS], Phys. Rev. C 98 (2018) no.2, 024908 doi:10.1103/PhysRevC.98.024908 [arXiv:1805.05424 [nucl-ex]].

[40] A. M. Sirunyan et al. [CMS], JHEP 05 (2018), 006 doi:10.1007/JHEP05(2018)006 [arXiv:1803.00042 [nucl-ex]].

[41] S. Chatrchyan et al. [CMS], Phys. Rev. C 90 (2014) no.2, 024908 doi:10.1103/PhysRevC.90.024908 [arXiv:1406.0932 [nucl-ex]].

[42] M. Aaboud et al. [ATLAS], Eur. Phys. J. C 77 (2017) no.6, 379 doi:10.1140/epjc/s10052-0174915-5 [arXiv:1702.00674 [hep-ex]].

[43] ALICE figure repository https://alice-figure.web.cern.ch/grp_prel_fig_pub

[44] G. Aad et al. [ATLAS], Phys. Rev. Lett. 124 (2020) no.22, 222002 doi:10.1103/PhysRevLett.124.222002 [arXiv:2004.03540 [hep-ex]]. 